\title{
Numerical simulation of the reinforced concrete barrier destruction at impact
}

\begin{abstract}
The given paper considers penetration of ogival projectile made from titanium and filled with the explosive substance into massive reinforced concrete barrier. Numerical simulation is three-dimensional conducted by means of EFES program software developed by the authors. Mathematical models take into account plastic properties of materials, and fracture. Projectile penetration has been considered as a surface normal, and under angle with the initial velocities of 300 and $700 \mathrm{~m} / \mathrm{s}$. Fracture patterns of barrier and explosive substance have been obtained with regards to the interaction conditions.
\end{abstract}

Volume I Issue 3 - 2017

PA Radchenko, SP Batuev, AV Radchenko
Tomsk State University of Architecture and Building, Russia

Correspondence: AV Radchenko, Tomsk State University of Architecture and Building, 2 Solyanaya Sq, Tomsk 634003, Russia, Email andrey-radchenko@live.ru

Received: July 19, 2017| Published: October 16, 2017

\section{Introduction}

Recent authors' works describe results of simulation of Boeing 747 interaction with protective shell ${ }^{1}$ in that case contact interaction of airplane body with the shell has been replaced by impulse. Such approach is justified when initial stage of interaction is described, taking that concrete structure contacts quite thin aluminum body (from 3 to $8 \mathrm{~mm}$ thick). In this case one does not need to calculate contact boundaries thus simplifying significantly the objective and enabling obtaining the results in short terms. When projectile impacts concrete barrier it is crucial to consider contact interaction, deformation and fracture of both barrier material and projectile material. Reinforced concrete structures used for nuclear power stations protection are generally designed with quite complex scheme of reinforcement. Modeling of projectiles penetration into such structures can be held by means of two approaches depending on the reinforcement design, size of reinforcing elements and the assigned objectives. The first approach suggests clear separation of reinforcing elements and therefore one need to calculate multiple contact boundaries thus complicating the given objective solution. The second approach is based on separation of locations in reinforced concrete structure which are adjacent to areas of reinforcement placement where material is described by mixture model. In this case there is only one contact boundary which is between the projectile and the barrier. The first approach can find application when it is necessary to obtain information on the influence of boundaries on shock-wave processes, to evaluate the adhesion properties of contact surfaces. However when assessment of integral parameters is required, second approach is appropriate to be applied. The given work contains calculations using second approach.

\section{Mathematical model}

The set of equations describing non-stationary adiabatic motions of compressible medium in general coordinates $(i=1,2,3)$, includes the following equations: ${ }^{2}$

a. Continuity equation

$$
\frac{\partial \rho}{\partial t}+\rho \nabla_{i} v^{i}=0
$$

b. Motion equation

$$
\rho a^{k}=\nabla_{i} \sigma^{\mathrm{ik}}+F^{k}
$$

Where

$$
\begin{aligned}
& a^{k}=\frac{\partial v^{k}}{\partial t}+v^{i} \nabla_{i} v^{k}, \\
& \nabla_{i} \sigma^{i k}=\sigma_{i}^{i k} \Gamma_{i m} k \sigma^{i m}+\Gamma_{i m}^{m} \sigma^{i k},
\end{aligned}
$$

c. Energy equation

$$
\frac{d E}{d t}=\frac{1}{\rho} \sigma^{\mathrm{ij}} e_{\mathrm{ij}}
$$

Where $F^{k}$ is components of mass force vector; $\Gamma_{\mathrm{ij}}^{k}$ - Christoffel symbols; $\sigma^{\mathrm{ij}}$ - contravariant components of symmetric stress tensor; $E$ - specific internal energy; $e_{\mathrm{ij}}$ - components of symmetric strain velocity tensor: $\rho$ - density of medium; $\bar{v}$ - velocity vector; $a^{k}$ components of acceleration vector;

$$
e_{\mathrm{ij}}=\frac{1}{2}\left(\nabla_{i} v_{j}+\nabla_{j} v_{i}\right)
$$

Behavior of materials under study both metal and concrete has been described by elasto-plastic model.

Stress tensor is presented as a sum of deviatoric $S^{\mathrm{ki}}$ and spherical part $P$ :

$$
\sigma^{i j}=-P g^{i j}+S^{i j},
$$

where $g^{i j}$ - metric tensor. Pressure inside the materials has been calculated using Mie-Gruneisen equation as a function of specific internal energy $E$ and density $\rho$ :

$$
P=\sum_{n=1}^{3} K_{n}\left(\frac{V}{V_{0}}-1\right)^{n}\left[1-K_{0}\left(\frac{V}{V_{0}}-1\right) / 2\right]+K_{0} \rho E,
$$

where $K_{0}, K_{1}, K_{2}, K_{3}$ - material constants, $V_{0}$ - initial specific volume, $V$ - current specific volume.

Suppose that the principle of minimum work of true stresses on the increments of plastic deformations is true for the medium, than the connection of component of strain velocity tensor and stress deviator is as follows: 


$$
2 G\left(g^{i m} g^{j k} e_{m k}-\frac{1}{3} g^{m k} e_{m k} g^{i j}\right)=\frac{D S^{i j}}{D t}+\lambda S^{i j},(\lambda \geq 0),
$$

In this case time derivatives of stress tensor are accepted by Jaumann definition:

$$
\frac{D S^{i j}}{D t}=\frac{d S^{i j}}{d t}-g^{i m} \omega_{m k} S^{k j}-g^{j m} \omega_{m k} S^{i k},
$$

where $\omega_{\mathrm{ij}}=\frac{1}{2}\left(\nabla_{i} v_{j}-\nabla_{j} v_{i}\right), G$ - shear modulus.

Consider that material behaves in elastic manner $(\lambda=0)$, in case when Von Mises criterion is followed:

$$
S^{\mathrm{ij}} S_{\mathrm{ij}} \leq \frac{2}{3} \sigma_{d}^{2},
$$

and it behaves in plastic manner $(\lambda>0)$, when the criterion is not followed. Here $\sigma_{d}$ - dynamic tensile yield stress that can in the general case be the function of deformations velocity, pressure and temperature. The dependency of yield stress on the pressure has been considered for concrete: ${ }^{3}$

$$
\sigma_{d}=7.7+\frac{11.398 P}{13.9+0.82 P}
$$

In case the condition (8) is violated, we apply the procedure of correction of stresses considering the material plasticity for calculation of the component of stress deviator. Components of $S^{\mathrm{ij}}$ are multiplied by normalizing factor, that equals to description of medium behavior in plastic zone as proved by equations of Prandtl-Reuss.

Limiting value of plastic strain intensity is accepted as a local criterion of shear fracture in metals:

$$
e_{u}=\frac{\sqrt{2}}{3} \sqrt{3 T_{2}-T_{1}^{2}}
$$

Where $\mathrm{T}_{1}, \mathrm{~T}_{2}$-first and second invariants of strain tensors.

To describe concrete fracture we use Hoffman criterion. ${ }^{4}$ The criterion considers the differences in ultimate tensile and compressive strength:

$$
\begin{aligned}
& C_{1}\left(\sigma_{22}-\sigma_{33}\right)^{2}+C_{2}\left(\sigma_{33}-\sigma_{11}\right)^{2}+C_{3}\left(\sigma_{11}-\sigma_{22}\right)^{2}+ \\
& +C_{4} \sigma_{11}+C_{5} \sigma_{22}+C_{6} \sigma_{33}+C_{7} \sigma_{12}^{2}+C_{8} \sigma_{23}^{2}+C_{9} \sigma_{31}^{2} \geq 1,
\end{aligned}
$$

where $C_{i}$ is defined from the following formulas:

$$
\begin{aligned}
& C_{1}=\left[\left(Y_{t} Y_{c}\right)^{-1}+\left(Z_{t} Z_{c}\right)^{-1}-\left(X_{t} X_{c}\right)^{-1}\right] / 2 ; \\
& C_{2}=\left[\left(X_{t} X_{c}\right)^{-1}+\left(Z_{t} Z_{c}\right)^{-1}-\left(Y_{t} Y_{c}\right)^{-1}\right] / 2 ; \\
& C_{3}=\left[\left(X_{t} X_{c}\right)^{-1}+\left(Y_{t} Y_{c}\right)^{-1}-\left(Z_{t} Z_{c}\right)^{-1}\right] / 2 ; \\
& C_{4}=\left(X_{t}^{-1}-X_{c}^{-1}\right) ;
\end{aligned}
$$

$$
\begin{aligned}
& C_{5}=\left(Y_{t}^{-1}-Y_{c}^{-1}\right) ; \\
& C_{6}=\left(Z_{t}^{-1}-Z_{c}^{-1}\right) ; \\
& C_{7}=S_{\mathrm{yz}}^{-2} ; \\
& C_{8}=S_{\mathrm{zx}}{ }^{-2} ; \\
& C_{9}=S_{\mathrm{xy}}{ }^{-2} .
\end{aligned}
$$

Where $X_{t}, X_{c}, Y_{t}, Y_{c}, Z_{t}, Z_{c}-\quad$ ultimate strength along axes $X, Y, Z$ under tension and compression respectively, while $S_{\mathrm{xy}}, S_{\mathrm{yz}}, S_{\mathrm{zx}}$-ultimate shear strength along the corresponding axes. In case of isotropic material $X_{t}=Y_{t}=Z_{t}=R_{t}, X_{c}=Y_{c}=Z_{c}=R_{c}$, $S_{\mathrm{xy}}=S_{\mathrm{yz}}=S_{\mathrm{zx}}=R_{s}$.

It is suggested that concrete fracture in conditions of intensive dynamic loads occurs the following way: ${ }^{1}$

In case strength criterion (11) is violated under conditions of compression $\left(e_{\mathrm{kk}} \leq 0\right)$, than further material behavior is described by hydrodynamic model, in that case concrete can be destructed when satisfying the criterion considering the ultimate value of plastic deformations intensity (10);

In case criterion (11) is violated under conditions of tension $\left(e_{\mathrm{kk}}>0\right)$, than material is considered to be completely destructed and components of stress tensor are supposed to be equal to zero.

Experiments have shown that dynamic loads lead to increasing strength properties of concrete. ${ }^{5}$ Moreover the dependency of ultimate tensile and compressive strength differs. Connection of static and dynamic ultimate strength is expressed by means of dynamic response factor:

$$
K_{d}=R_{d} / R_{s}
$$

where $R_{d}$ - dynamic strength, $R_{s}$ - static strength.

Based on the experimental data dynamic response factor has been obtained for concrete under compression (12) and tension (13). Corresponding curves are given in Figure 1, where curve 1 describes dependency of dynamic response factor under tension on deformations velocity, curve 2 describes dependency under compression.

$$
\begin{aligned}
& K_{\mathrm{dt}}=0.00158333 e^{5}+0.0252855 e^{4} \\
& \quad+0.15255 e^{3}+0.47898 e^{2}+1.01959 e+2.36037 . \\
& K_{\mathrm{dc}}=0.000832308 e^{5}+0.0110547 e^{4}+ \\
& +0.0447734 e^{3}+0.0475887 e^{2}+0.0184316 e+1.20895 .
\end{aligned}
$$

Ultimate shear strength of concrete is defined from the values of ultimate compressive and tensile strength: ${ }^{3}$

$$
R_{s}=0.55 \sqrt{R_{c} R_{t}}
$$


Important aspect while numerical modeling of impact interaction is selecting the algorithm of contact boundaries calculation. Generally, the existing program software use algorithms "element-node" and "node-node" to define the possible penetration of one body into another. The given work suggests the algorithm of "elementelement" type, ${ }^{6}$ proved oneself to be appropriate while solving the three dimensional objectives and enabling to use possibilities of parallel computing to a maximum extent.

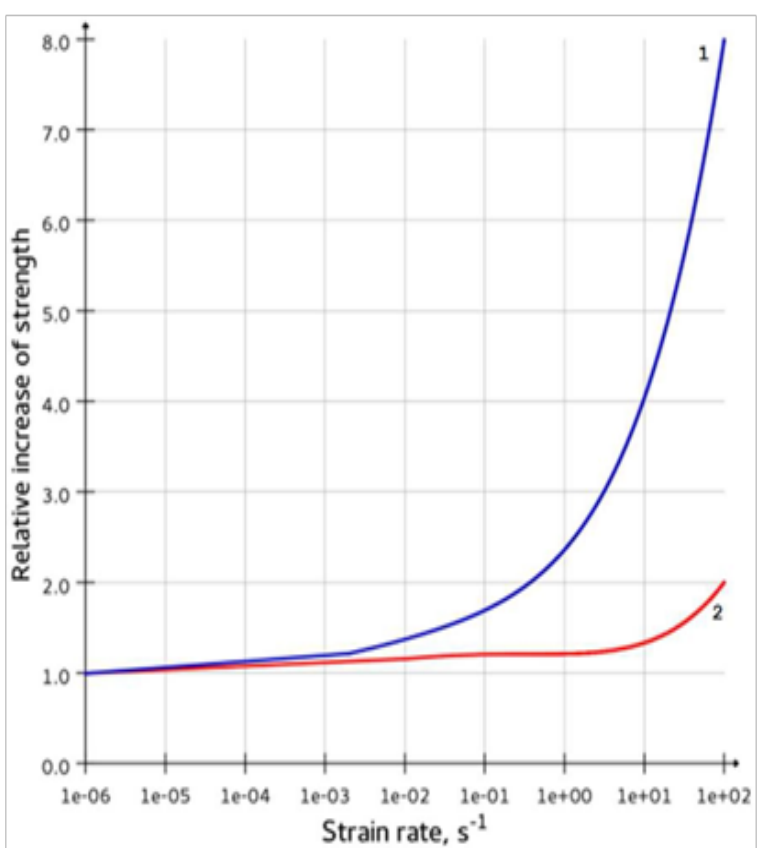

Figure I Dependency of ultimate strength of concrete on deformation velocity: I - under tension, 2 -under compression.

\section{Numerical results}

The results of numerical simulation of ogival projectile interaction with reinforced concrete barrier are given below. The properties of the investigated materials are given in Table 1 . The projective is given as

Table I Properties of materials a shell made from titanium and filled with explosive substance. The thickness of reinforced concrete barrier is $1 \mathrm{~m}$. Layers of reinforcement are placed close to front and back surfaces of the barrier. The initial projectile velocity is 300 and $700 \mathrm{~m} / \mathrm{s}$. Two types of interaction have been considered: the standard one, and under the angle of 5 degrees to normal line.

Figure 2 shows in section the standard penetration of projectile with initial velocity of $700 \mathrm{~m} / \mathrm{s}$. The areas of reinforcement in the barrier are marked in red color. Through penetration of barrier and concrete fracture has been observed. Concrete is destructed due to the action of tensile stresses. Because of low tensile strength of concrete it is destructed almost through the entire surface. One can also note destruction of explosive substance close to the projectile nose in the central area. Destruction of explosive substance is conditioned by the action of unloading wave distributed from the lateral surface of projectile. In this case titanium shell is deformed insignificantly almost completely saving its initial shape.

Figure 3 shows normal penetration of projectile with the velocity of $300 \mathrm{~m} / \mathrm{s}$. In general the fracture pattern is similar to Figure 2. Larger level of destruction in the barrier and the explosive substance is conditioned by longer process of interaction thus it has lead to the moment of barrier striking (Figure 3C) and larger damage cumulation.

(Figure 4) (Figure 5C) show computation configuration of the projectile and barrier by interaction under angle of 5 degrees for the initial velocities of 300 and $700 \mathrm{~m} / \mathrm{s}$, correspondently. The same as for normal interaction (Figure 2) (Figure 3) one can observe consistent reinforced concrete barrier striking. When impacting under angle localization and geometry of destruction areas as well as destruction in explosive substance is changed. The areas of destruction in the explosive substance are formed not only in the central part of the projectile but also close to the titanium shell in the nose part on the right (Figure 4B) (Figure 5B). This can be explained by the fact that during impact under angle this part of projectile is free at the initial moment of interaction, thus the unloading wave is being formed here, further leading to tensile stresses occurrence which destruct explosive substance.

\begin{tabular}{lllllll}
\hline Material & $\tilde{\mathbf{n}} \mathbf{, ~} \mathbf{k g} / \mathbf{m}^{\mathbf{3}}$ & $\begin{array}{l}\text { Speed of sound } \\
\mathbf{C}, \mathbf{m} / \mathbf{s}\end{array}$ & $\begin{array}{l}\text { Poisson's } \\
\text { ratio }\end{array}$ & $\begin{array}{l}\text { Tensile strength, } \\
\mathbf{M P a}\end{array}$ & $\begin{array}{l}\text { Compressive } \\
\text { strength, MPa }\end{array}$ & $\begin{array}{l}\text { Young's modulus } \\
\mathrm{E}, \mathbf{G p a}\end{array}$ \\
\hline Concrete & 2450 & 4500 & 0,2 & 1,75 & 22 & 26 \\
Titanium & 4500 & 6900 & 0.32 & 400 & 400 & 146 \\
Steel & 7850 & 5930 & 0,3 & 400 & 400 & 204 \\
\hline
\end{tabular}

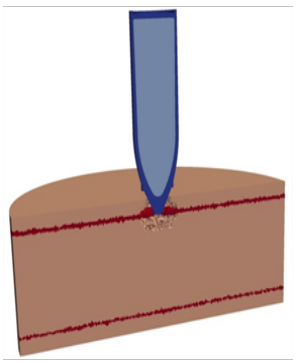

a)

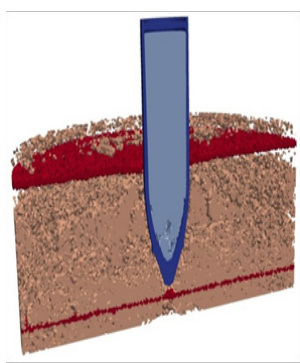

b)

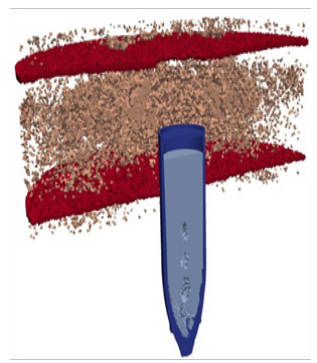

c)

Figure 2 Configuration of projectile and barrier. $v_{0}=700 \mathrm{~m} / \mathrm{s}, \alpha=0^{\circ}$ degrees. (A) $\mathrm{t}=0.2 \mathrm{~ms}$, (B) $\mathrm{t}=1.2 \mathrm{~ms},(\mathrm{C}) \mathrm{t}=3 \mathrm{~ms}$. 


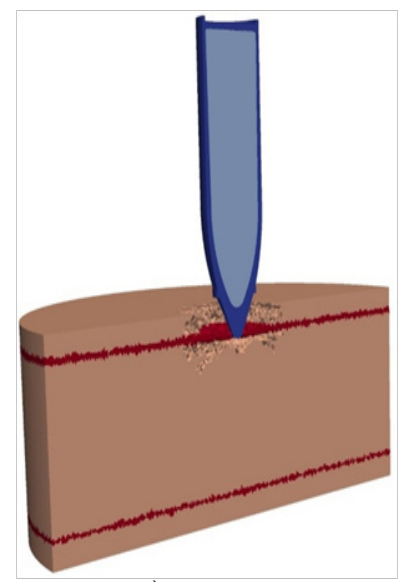

a)

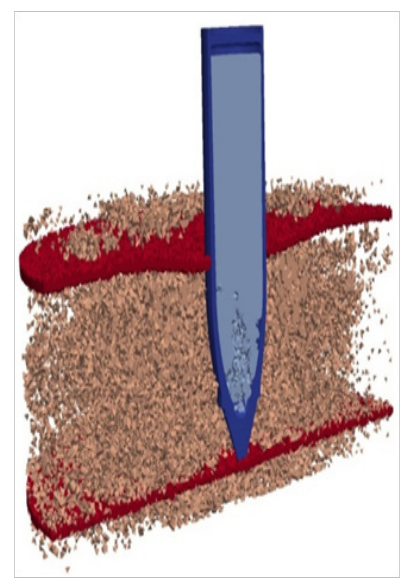

b)

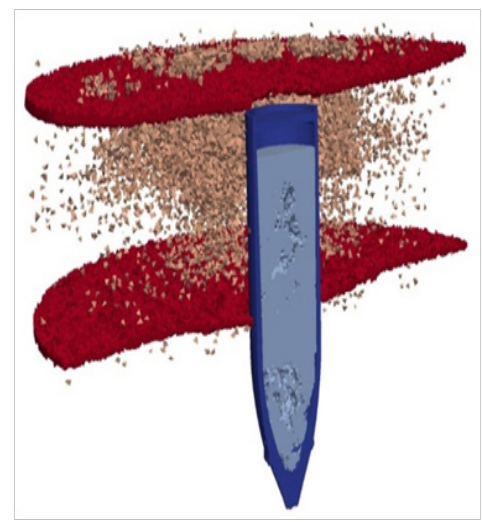

c)

Figure 3 Configuration of projectile and barrier. $v_{0}=300 \mathrm{~m} / \mathrm{s}, \alpha=0^{\circ}$. (A) $\mathrm{t}=0.5 \mathrm{~ms}$, (B) $\mathrm{t}=3 \mathrm{~ms}$, (C) $\mathrm{t}=6 \mathrm{~ms}$

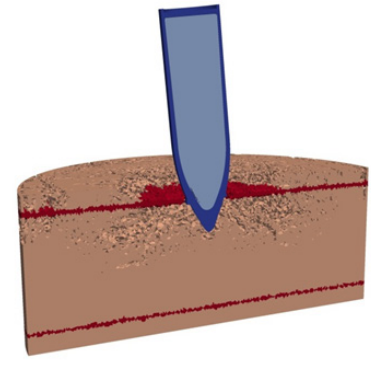

a)

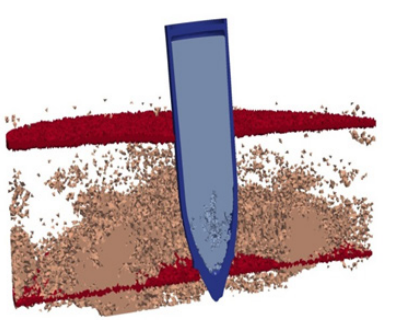

b)
Figure 4 Configurations of projectile and barrier. $v_{0}=700 \mathrm{~m} / \mathrm{s}, \alpha=5^{\circ}$. Time periods (A) $t=0.5 \mathrm{~ms}$, (B) $t=1.5 \mathrm{~ms}$.
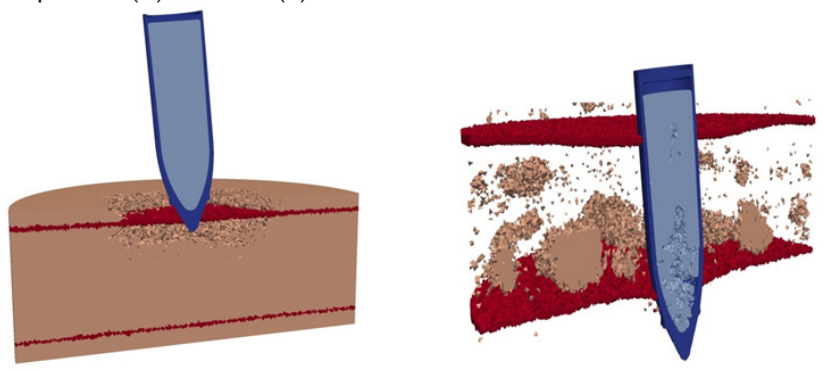

a)

b)

Figure 5 Configurations of projectile and barrier. $v_{0}=300 \mathrm{~m} / \mathrm{s}, \alpha=5^{\circ}$. (A) $\mathrm{t}=0.7 \mathrm{~ms}$, (B) $\mathrm{t}=4.6 \mathrm{~ms}$

\section{Conclusion}

Resulting from the conducted studies the following conclusions can be made:

A. The model has been suggested for reinforced concrete barriers behavior under impact.

The conditions of interaction on the projectile and reinforced concrete barrier destruction have been studied.

Wide-parameter numerical studies on selection of optimal structural solutions are possible to be conducted based on the developed methodology.

\section{Acknowledgments}

The work has been conducted with the financial support of the President of the Russian Federation No. MK-413.2017.1.

\section{Conflicts of interest}

Author declares that there is no conflict of interest.

\section{References}

1. Radchenko PA, Batuev SP, Radchenko AV, et al. Numerical simulation of deformation and fracture of space protective shell structures from concrete and fiber concrete under pulse loading. J Phys Conf Ser. 2015;653(1):012048.

2. Radchenko PA, Batuev S P, Radchenko AV, et al. Destruction of concrete beams with metal and composite reinforcement under impulse action. J Phys Conf Ser. 2015;653(1)012047.

3. Belov NN, Kabantsev OV, Kopanitsa DG, et al. Experimental analysis of dynamic strength of reinforced concrete structures elements. 2008.

4. Wu EM. Mechanics of Composite Materials. 1978. p. 401-491.

5. International Atomic Energy Agency Safety aspects of nuclear power plants against human induced external events: Assessment of structures Draft Safety Report.

6. Radchenko PA. Tambov Univ Rep Ser Nat and Tech Sc. 2016;21:1275-1287. 